\title{
NONZERO POSITIVE SOLUTIONS OF NONLOCAL ELLIPTIC SYSTEMS WITH FUNCTIONAL BCS
}

\author{
GENNARO INFANTE
}

\begin{abstract}
We discuss the existence and non-existence of non-negative weak solutions for second order nonlocal elliptic systems subject to functional boundary conditions. Our approach is based on classical fixed point index theory combined with some recent results by the author.
\end{abstract}

\section{INTRODUCTION}

There has been growing attention to the solvability of elliptic equations where nonlocal terms occur, one motivation being that these kind of equations often occur in applications. A widely studied case is the one of Kirchoff-type equations, see for example the review by Ma [31]. Under Dirichlet boundary conditions (BCs) the equation

$$
-a\left(\int_{\Omega}|u|^{p} d x\right) \Delta u=\lambda f(x, u), x \in \Omega
$$

has been studied, for example, by Corrêa and co-authors [11, 12], Jiang and Zhai [26] and by Yan and co-authors [43, 44, 45]. Here $\Omega \subset \mathbb{R}^{m}, m \geq 1$, is a domain with sufficiently smooth boundary, $a$ is a positive function, $p \in \mathbb{R}$.

Note that in the local framework (that corresponds to $a \equiv 1$ in (1.1)) one regains the classical Gelfand-type problem

$$
-\Delta u=\lambda f(x, u), x \in \Omega,\left.\quad u\right|_{\partial \Omega}=0,
$$

we refer to the Introduction of [3] for a recent review on this topic.

By means of the classical Krasnosel'skil-Guo fixed-point theorem Stańczy [38], when $\Omega$ is a ball or an annular domain, studied the equation

$$
-\left(\int_{\Omega} f(u) d x\right)^{p} \Delta u=\lambda(f(u))^{q}, x \in \Omega,
$$

a setting that covers, for example, the celebrated mean field equation

$$
-\Delta u=\lambda \frac{e^{u}}{\int_{\Omega} e^{u} d x}, x \in \Omega,
$$

2010 Mathematics Subject Classification. Primary 35J47, secondary 35B09, 35J57, 35J60, 47H10.

Key words and phrases. Positive solution, nonlocal elliptic system, functional boundary condition, cone, fixed point index. 
we refer the reader to [9, 15] for further details on the equation (1.2) in dimension 2.

Arcoya and co-authors [2], by means of a Bolzano theorem, studied the equation

$$
-\left(\int_{\Omega} g(u) d x\right)^{p} \Delta u=f(u), x \in \Omega,
$$

while Corrêa and de Morais Filho [13] studied the equation

$$
-\left(\int_{\Omega} g(x, u) d x\right)^{p} \Delta u=(f(x, u))^{q}, x \in \Omega
$$

via the Galerkin method.

In the radial case, also by topological methods, Fijałkowski and Przeradzki [17] and Enguiça and Sanchez [14] studied the equation

$$
-\Delta u=f\left(u, \int_{\Omega} g(u) d x\right)
$$

note that in (1.3) a nonlocal term occurs within the nonlinearity $f$, in what follows we shall consider a similar setting.

It is worth to mention that the fairly general equation

$$
-\mathcal{A}(x, u) \Delta u=\lambda f(u), x \in \Omega
$$

where $\mathcal{A}$ is a suitable functional defined on $\Omega \times L^{p}(\Omega)$, has been studied by Chipot and co-authors [6, 7], while Faraci and Iannizzotto [16] studied the equation

$$
-\Delta u-\mu_{1} u=A[u] f(u), x \in \Omega,
$$

where $\mu_{1}$ is the principal eigenvalue of the Laplacian with Dirichlet BCs and $A[u]$ is a nonlinear functional. The interesting case of $p$-Laplacian equations with nonlocal terms has been recently discussed by Santos and co-authors in [36, 37].

The common feature of the above mentioned problems is the requirement that the solution vanishes on the boundary of $\Omega$. Non-homogeneous BCs in the case of nonlocal elliptic equations have been recently studied by Wang and An [40] and Morbach and Corrẽa [32].

In the context of systems of nonlocal elliptic equations, we mention the papers by Chen and Gao [5] and the recent paper by do Ó et al [30]. In particular in the latter paper the authors study, in the radial case and by topological methods, the system

$$
\left\{\begin{array}{ccc}
-a_{i}\left(\int_{\Omega}\left|u_{i}\right|^{p_{i}} d x\right) \Delta u_{i}=\lambda_{i} f_{i}(|x|, u), & x \in \Omega, & i=1,2, \ldots, n \\
u_{i}=0, & x \in \partial \Omega, & i=1,2, \ldots, n
\end{array}\right.
$$

Here we adapt the arguments of [23], valid for local differential equations, in order to study the solvability of the system of second order elliptic functional differential equations subject 
to functional BCs

$$
\left\{\begin{array}{ccc}
L_{i} u_{i}=\lambda_{i} f_{i}\left(x, u, w_{i}[u]\right), & x \in \Omega, & i=1,2, \ldots, n, \\
B_{i} u_{i}=\eta_{i} \zeta_{i}(x) h_{i}[u], & x \in \partial \Omega, & i=1,2, \ldots, n,
\end{array}\right.
$$

where $\Omega \subset \mathbb{R}^{m}(m \geq 2)$ is a bounded domain with a sufficiently smooth boundary, $L_{i}$ is a strongly uniformly elliptic operator, $B_{i}$ is a first order boundary operator, $u=\left(u_{1}, \ldots, u_{n}\right)$, $f_{i}$ are continuous functions, $\zeta_{i}$ are sufficiently regular functions, $w_{i}, h_{i}$ are suitable compact functionals, $\lambda_{i}, \eta_{i}$ are nonnegative parameters. The setting for the BCs covers, for example, the special cases of linear (multi-point or integral) BCs of the form

$$
h_{i}[u]=\sum_{k=1}^{n} \sum_{j=1}^{N} \hat{\alpha}_{i j k} u_{k}\left(\omega_{j}\right), \text { or } h_{i}[u]=\sum_{k=1}^{n} \int_{\Omega} \hat{\alpha}_{i k}(x) u_{k}(x) d x,
$$

where $\hat{\alpha}_{i j k}$ are non-negative coefficients, $\omega_{j} \in \Omega, \hat{\alpha}_{i k}$ are non-negative continuous functions on $\bar{\Omega}$. There exists a wide literature on these kind of BCs, we refer the reader to the reviews [4, 10, 29, 33, 39, 42] and the papers [24, 25, 34, 35, 41]. We point that that this setting can also be applied to nonlinear, nonlocal BCs, which have seen recently attention in the framework of (local) elliptic equations, we refer the reader to the papers by Cianciaruso and co-authors [8] and Goodrich [18, 19, 20, 21].

Here we discuss, under fairly general conditions, the existence and non-existence of positive solutions of the system (1.4). Our approach relies on classical fixed point index theory. We present some applications of the theoretical results to nonlocal elliptic systems, where we illustrate the variety of BCs that can be approached via this method. Our results are new and complement the results of [30], by considering non-radial cases, by allowing the presence of functional BCs and by permitting, in the non-local terms of differential equations, an interaction between all the components of the system. We also improve the results in [23] in the case of local elliptic equations, by weakening the assumptions on the BCs.

\section{EXISTENCE AND NON-EXISTENCE RESUlts}

In what follows, for every $\hat{\mu} \in(0,1)$ we denote by $C^{\hat{\mu}}(\bar{\Omega})$ the space of all $\hat{\mu}$-Hölder continuous functions $g: \bar{\Omega} \rightarrow \mathbb{R}$ and, for every $k \in \mathbb{N}$, we denote by $C^{k+\hat{\mu}}(\bar{\Omega})$ the space of all functions $g \in C^{k}(\bar{\Omega})$ such that all the partial derivatives of $g$ of order $k$ are $\hat{\mu}$-Hölder continuous in $\bar{\Omega}$ (for more details see [1, Examples 1.13 and 1.14]).

We make the following assumptions on the domain $\Omega$ and the operators $L_{i}$ and $B_{i}$ and the functions $\zeta_{i}$ that occur in (1.4) (see [1, Section 4 of Chapter 1] and [27, 28])):

(1) $\Omega \subset \mathbb{R}^{m}, m \geq 2$, is a bounded domain such that its boundary $\partial \Omega$ is an $(m-1)$ dimensional $C^{2+\hat{\mu}}$-manifold for some $\hat{\mu} \in(0,1)$, such that $\Omega$ lies locally on one side of $\partial \Omega$ (see [46, Section 6.2] for more details). 
(2) $L_{i}$ is a the second order elliptic operator given by

$L_{i} u(x)=-\sum_{j, l=1}^{m} a_{i j l}(x) \frac{\partial^{2} u}{\partial x_{j} \partial x_{l}}(x)+\sum_{j=1}^{m} a_{i j}(x) \frac{\partial u}{\partial x_{j}}(x)+a_{i}(x) u(x), \quad$ for $x \in \Omega$,

where $a_{i j l}, a_{i j}, a_{i} \in C^{\hat{\mu}}(\bar{\Omega})$ for $j, l=1,2, \ldots, m, a_{i}(x) \geq 0$ on $\bar{\Omega}, a_{i j l}(x)=a_{i j l}(x)$ on $\bar{\Omega}$ for $j, l=1,2, \ldots, m$. Moreover $L_{i}$ is strongly uniformly elliptic; that is, there exists $\bar{\mu}_{i 0}>0$ such that

$$
\sum_{j, l=1}^{m} a_{i j l}(x) \xi_{j} \xi_{l} \geq \bar{\mu}_{i 0}\|\xi\|^{2} \quad \text { for } x \in \Omega \text { and } \xi=\left(\xi_{1}, \xi_{2}, \ldots, \xi_{m}\right) \in \mathbb{R}^{m} .
$$

(3) $B_{i}$ is a boundary operator given by

$$
B_{i} u(x)=b_{i}(x) u(x)+\delta_{i} \frac{\partial u}{\partial \nu}(x) \quad \text { for } x \in \partial \Omega,
$$

where $\nu$ is an outward pointing and nowhere tangent vector field on $\partial \Omega$ of class $C^{1+\hat{\mu}}$ (not necessarily a unit vector field), $\frac{\partial u}{\partial \nu}$ is the directional derivative of $u$ with respect to $\nu, b_{i}: \partial \Omega \rightarrow \mathbb{R}$ is of class $C^{1+\hat{\mu}}$ and moreover one of the following conditions holds:

(a) $\delta_{i}=0$ and $b_{i}(x) \equiv 1$ (Dirichlet boundary operator).

(b) $\delta_{i}=1, b_{i}(x) \equiv 0$ and $a_{i}(x) \not \equiv 0$ (Neumann boundary operator).

(c) $\delta_{i}=1, b_{i}(x) \geq 0$ and $b_{i}(x) \not \equiv 0$ (Regular oblique derivative boundary operator). (4) $\zeta_{i} \in C^{2-\delta_{i}+\hat{\mu}}(\partial \Omega)$.

Under the previous conditions (see [1], Section 4 of Chapter 1) a strong maximum principle holds, given $g \in C^{\hat{\mu}}(\bar{\Omega})$, the BVP

$$
\begin{cases}L_{i} u(x)=g(x), & x \in \Omega, \\ B_{i} u(x)=0, & x \in \partial \Omega,\end{cases}
$$

admits a unique classical solution $u \in C^{2+\hat{\mu}}(\bar{\Omega})$ and, moreover, given $\zeta_{i} \in C^{2-\delta_{i}+\hat{\mu}}(\partial \Omega)$ the BVP

$$
\begin{cases}L_{i} u(x)=0, & x \in \Omega, \\ B_{i} u(x)=\zeta_{i}(x), & x \in \partial \Omega,\end{cases}
$$

also admits a unique solution $\gamma_{i} \in C^{2+\hat{\mu}}(\bar{\Omega})$.

We recall that a cone $P$ of a real Banach space $X$ is a closed set with $P+P \subset P, \lambda P \subset P$ for all $\lambda \geq 0$ and $P \cap(-P)=\{0\}$. A cone $P$ induces a partial ordering in $X$ by means of the relation $x \leq y$ if and only if $y-x \in P$. The cone $P$ is normal if there exists $d>0$ such that for all $x, y \in X$ with $0 \leq x \leq y$ then $\|x\| \leq d\|y\|$. Note that every cone $P$ has the Archimedean property; that is, $n x \leq y$ for all $n \in \mathbb{N}$ and some $y \in X$ implies $x \leq 0$. In what follows, with abuse of notation, we will use the same symbol " $\geq$ " for the different cones appearing in the paper. 
In order to seek solutions of the system (1.4), we make use of the cone of non-negative functions $P=C\left(\bar{\Omega}, \mathbb{R}_{+}\right)$. The solution operator associated to the BVP (2.1), $K_{i}: C^{\hat{\mu}}(\bar{\Omega}) \rightarrow$ $C^{2+\hat{\mu}}(\bar{\Omega})$, defined as $K_{i} g=u$ is linear and continuous. It is known (see [1], Section 4 of Chapter 1) that $K_{i}$ can be extended uniquely to a continuous, linear and compact operator (that we denote again by the same name) $K_{i}: C(\bar{\Omega}) \rightarrow C(\bar{\Omega})$ that leaves the cone $P$ invariant, that is $K_{i}(P) \subset P$. We denote by $r\left(K_{i}\right)$ the spectral radius of $K_{i}$. It is known (for details see Lemma 3.3 of [28]) that $r\left(K_{i}\right) \in(0,+\infty)$ and there exists $\varphi_{i} \in P \backslash\{0\}$ such that

$$
\varphi_{i}=\mu_{i} K_{i} \varphi_{i}
$$

where $\mu_{i}=1 / r\left(K_{i}\right)$.

We utilize the space $C\left(\bar{\Omega}, \mathbb{R}^{n}\right)$, endowed with the norm $\|u\|:=\max _{i=1,2, \ldots, n}\left\{\left\|u_{i}\right\|_{\infty}\right\}$, where $\|z\|_{\infty}=\max _{x \in \bar{\Omega}}|z(x)|$, and consider (with abuse of notation) the cone $P=C\left(\bar{\Omega}, \mathbb{R}_{+}^{n}\right.$ ). Given $J=\prod_{i=1}^{n} J_{i} \subset \mathbb{R}_{+}^{n}$, where each $J_{i} \subset \mathbb{R}$ is a closed nonempty interval, we define

$$
P_{J}=\{u \in P: u(x) \in J \text { for all } x \in \bar{\Omega}\} .
$$

We now fix $I=\prod_{i=1}^{n}\left[0, \rho_{i}\right]$, where $\rho_{i} \in(0,+\infty)$, and rewrite the elliptic system (1.4) as a fixed point problem by considering the operators $T, \Gamma: C(\bar{\Omega}, I) \rightarrow C\left(\bar{\Omega}, \mathbb{R}^{n}\right)$ given by

$$
T(u):=\left(\lambda_{i} K_{i} F_{i}(u)\right)_{i=1 . . n}, \quad \Gamma(u):=\left(\eta_{i} \gamma_{i} h_{i}[u]\right)_{i=1 . . n},
$$

where $K_{i}$ is the above mentioned extension of the solution operator associated to (2.1), $\gamma_{i} \in C^{2+\hat{\mu}}(\bar{\Omega})$ is the unique solution of the BVP (2.2) and

$$
F_{i}(u)(x):=f_{i}\left(x, u(x), w_{i}[u]\right), \text { for } u \in C(\bar{\Omega}, I) \text { and } x \in \bar{\Omega} \text {. }
$$

Definition 2.1. We say that $u \in C(\bar{\Omega}, J)$ is a weak solution of the system (1.4) if and only if $u$ is a fixed point of the operator $T+\Gamma$, that is,

$$
u=T u+\Gamma u=\left(\lambda_{i} K_{i} F_{i}(u)+\eta_{i} \gamma_{i} h_{i}[u]\right)_{i=1 . . n}
$$

if, furthermore, the components of $u$ are non-negative with $u_{j} \not \equiv 0$ for some $j$ we say that $u$ is a nonzero positive solution of the system (1.4).

For our existence result, we make use of the following Proposition that states the main properties of the classical fixed point index, for more details see [1, 22]. In what follows the closure and the boundary of subsets of a cone $\hat{P}$ are understood to be relative to $\hat{P}$.

Proposition 2.2. Let $X$ be a real Banach space and let $\hat{P} \subset X$ be a cone. Let $D$ be an open bounded set of $X$ with $0 \in D \cap \hat{P}$ and $\overline{D \cap \hat{P}} \neq \hat{P}$. Assume that $T: \overline{D \cap \hat{P}} \rightarrow \hat{P}$ is a compact operator such that $x \neq T x$ for $x \in \partial(D \cap \hat{P})$. Then the fixed point index $i_{\hat{P}}(T, D \cap \hat{P})$ has the following properties: 
(i) If there exists $e \in \hat{P} \backslash\{0\}$ such that $x \neq T x+\lambda$ for all $x \in \partial(D \cap \hat{P})$ and all $\lambda>0$, then $i_{\hat{P}}(T, D \cap \hat{P})=0$.

(ii) If $T x \neq \lambda x$ for all $x \in \partial(D \cap \hat{P})$ and all $\lambda>1$, then $i_{\hat{P}}(T, D \cap \hat{P})=1$.

(iii) Let $D^{1}$ be open bounded in $X$ such that $\left(\overline{D^{1} \cap \hat{P}}\right) \subset(D \cap \hat{P})$. If $i_{\hat{P}}(T, D \cap \hat{P})=1$ and $i_{\hat{P}}\left(T, D^{1} \cap \hat{P}\right)=0$, then $T$ has a fixed point in $(D \cap \hat{P}) \backslash\left(\overline{D^{1} \cap \hat{P}}\right)$. The same holds if $i_{\hat{P}}(T, D \cap \hat{P})=0$ and $i_{\hat{P}}\left(T, D^{1} \cap \hat{P}\right)=1$.

With these ingredients we can now state a result regarding the existence of positive solutions for the system (1.4), that extends the results of Theorem 2.4 of [23] to this new setting. In the sequel we denote by $\hat{1}$ the constant function equal to 1 on $\bar{\Omega}$.

Theorem 2.3. Let $I=\prod_{i=1}^{n}\left[0, \rho_{i}\right]$ and assume the following conditions hold.

(a) For every $i=1,2, \ldots, n, w_{i}: P_{I} \rightarrow \mathbb{R}$ is continuous,

$$
-\infty<\underline{w}_{i}:=\inf _{u \in P_{I}} w_{i}[u] \text { and } \bar{w}_{i}:=\sup _{u \in P_{I}} w_{i}[u]<+\infty \text {. }
$$

(b) For every $i=1,2, \ldots, n, f_{i} \in C\left(\bar{\Omega} \times I \times\left[\underline{w}_{i}, \bar{w}_{i}\right]\right)$ and $f_{i} \geq 0$. Set

$$
M_{i}:=\max _{(x, u, w) \in \bar{\Omega} \times I \times\left[\underline{w}_{i}, \bar{w}_{i}\right]} f_{i}(x, u, w) .
$$

(c) There exist $\delta \in(0,+\infty), i_{0} \in\{1,2, \ldots, n\}$ and $\rho_{0} \in\left(0, \min _{i=1 . . n} \rho_{i}\right)$ such that

$$
f_{i_{0}}(x, u, w) \geq \delta u_{i_{0}}, \text { for every }(x, u, w) \in \bar{\Omega} \times I_{0} \times\left[\underline{w}_{i_{0}}^{0}, \bar{w}_{i_{0}}^{0}\right],
$$

where $I_{0}:=\prod_{i=1}^{n}\left[0, \rho_{0}\right]$ and

$$
\underline{w}_{i_{0}}^{0}:=\inf _{u \in P_{I_{0}}} w_{i_{0}}[u] \leq \bar{w}_{i_{0}}^{0}:=\sup _{u \in P_{I_{0}}} w_{i_{0}}[u] .
$$

(d) For every $i=1,2, \ldots, n, \zeta_{i} \in C^{2-\delta_{i}+\hat{\mu}}(\partial \Omega), \zeta_{i} \geq 0, h_{i}: P_{I} \rightarrow[0,+\infty)$ is continuous and bounded. We set

$$
\bar{h}_{i}:=\sup _{u \in \partial P_{I}} h_{i}[u] .
$$

(e) For every $i=1,2, \ldots, n$ the following two inequalities are satisfied

$$
\frac{\mu_{i_{0}}}{\delta} \leq \lambda_{i_{0}} \text { and } \lambda_{i} M_{i}\left\|K_{i}(\hat{1})\right\|_{\infty}+\eta_{i} \bar{h}_{i}\left\|\gamma_{i}\right\|_{\infty} \leq \rho_{i} .
$$

Then the system (1.4) has a nonzero positive weak solution u such that

$$
\rho_{0} \leq\|u\| \text { and }\left\|u_{i}\right\|_{\infty} \leq \rho_{i}, \text { for every } i=1,2, \ldots, n \text {. }
$$

Proof. Due to the assumptions above the operator $T+\Gamma$ maps $P_{I}$ into $P$ and is compact (by construction, the map $F$ is continuous and bounded and $\Gamma$ is a finite rank operator). If $T+\Gamma$ has a fixed point on $\partial P_{I} \cup \partial P_{I_{0}}$ we are done. If $T+\Gamma$ is fixed point free on $\partial P_{I} \cup \partial P_{I_{0}}$, then the fixed point index is defined and we can make use of Proposition 2.2. 
We now show, by contradiction, that

$$
\sigma u \neq T u+\Gamma u \text { for every } u \in \partial P_{I} \text { and every } \sigma>1 \text {. }
$$

If this false, then there exist $u \in \partial P_{I}$ and $\sigma>1$ such that $\sigma u=T u+\Gamma u$. Since $u \in \partial P_{I}$ there exists $j$ such that $\left\|u_{j}\right\|_{\infty}=\rho_{j}$ and for every for every $x \in \bar{\Omega}$ we have

$$
\begin{aligned}
& \sigma u_{j}(x)=\lambda_{j} K_{j} F_{j}(u)(x)+\eta_{j} h_{j}[u] \gamma_{j}(x) \leq\left\|\lambda_{j} K_{j} F_{j}(u)+\eta_{j} h_{j}[u] \gamma_{j}\right\|_{\infty} \\
& \leq\left\|\lambda_{j} K_{j}\left(M_{j} \hat{1}\right)\right\|_{\infty}+\left\|\eta_{j} \bar{h}_{j} \gamma_{j}\right\|_{\infty}=\lambda_{j} M_{j}\left\|K_{j}(\hat{1})\right\|_{\infty}+\eta_{j} \bar{h}_{j}\left\|\gamma_{j}\right\|_{\infty} \leq \rho_{j} .
\end{aligned}
$$

Passing to the supremum for $x \in \bar{\Omega}$ in (2.5) we get $\sigma \rho_{j} \leq \rho_{j}$, a contradiction. By (ii) of Proposition 2.2 we obtain

$$
i_{P}\left(T+\Gamma, P_{I} \backslash \partial P_{I}\right)=1 .
$$

We now show, by contradiction, that

$$
u \neq T u+\Gamma u+\sigma \varphi \text { for every } u \in \partial P_{I_{0}} \text { and every } \sigma>0,
$$

where $\varphi=\left(\varphi_{1}, \ldots, \varphi_{n}\right)$ and $\varphi_{i}$ given by (2.3) .

If this does not hold, there exists $u \in \partial P_{\rho_{0}}$ and $\sigma>0$ such that $u=T u+\Gamma u+\sigma \varphi$. Then we have $u \geq \sigma \varphi$ and, in particular, $u_{i_{0}} \geq \sigma \varphi_{i_{0}}$. For every $x \in \bar{\Omega}$ we have

$$
\begin{aligned}
u_{i_{0}}(x)=\left(\lambda_{i_{0}} K_{i_{0}} F_{i_{0}} u\right)(x)+\eta_{i_{0}} h_{i_{0}}[u] \gamma_{i_{0}}(x)+\sigma \varphi_{i_{0}}(x) & \\
\geq\left(\lambda_{i_{0}} K_{i_{0}} \delta u_{i_{0}}\right)(x)+\sigma \varphi_{i_{0}}(x) \geq\left(\lambda_{i_{0}} \delta K_{i_{0}}\left(\sigma \varphi_{i_{0}}\right)\right)(x)+\sigma \varphi_{i_{0}}(x) & \\
& =\frac{\sigma \lambda_{i_{0}} \delta}{\mu_{i_{0}}} \varphi_{i_{0}}(x)+\sigma \varphi_{i_{0}}(x) \geq 2 \sigma \varphi_{i_{0}}(x) .
\end{aligned}
$$

By iteration we obtain, for $x \in \bar{\Omega}$,

$$
u_{i_{0}}(x) \geq n \sigma \varphi_{i_{0}}(x) \text { for every } n \in \mathbb{N},
$$

which contradicts the boundedness of $u$. This gives, by $(i)$ of Proposition 2.2, that

$$
i_{P}\left(T+\Gamma, P_{I_{0}} \backslash \partial P_{I_{0}}\right)=0 .
$$

By means of $(i i i)$ of Proposition 2.2, $T+\Gamma$ has a fixed point in $P_{I} \backslash\left(\partial P_{I} \cup P_{I_{0}}\right)$.

We now provide a non-existence result which extends Theorem 2.7 of [23].

Theorem 2.4. Let $I=\prod_{i=1}^{n}\left[0, \rho_{i}\right]$ and assume that for every $i=1,2, \ldots, n$ we have:

- $f_{i} \in C\left(\bar{\Omega} \times I \times\left[\underline{w}_{i}, \bar{w}_{i}\right]\right)$ and there exist $\tau_{i} \in(0,+\infty)$ such that

$$
0 \leq f_{i}(x, u, w) \leq \tau_{i} u_{i}, \text { for every }(x, u, w) \in \bar{\Omega} \times I \times\left[\underline{w}_{i}, \bar{w}_{i}\right]
$$

- $\zeta_{i} \in C^{2-\delta_{i}+\hat{\mu}}(\partial \Omega), \zeta_{i} \geq 0, h_{i}: P_{I} \rightarrow[0,+\infty)$ is continuous and there exist $\theta_{i} \in$ $(0,+\infty)$ and

$$
h_{i}[u] \leq \theta_{i}\|u\|, \underset{7}{\text { for every }} u \in P_{I} \text {, }
$$


- the following inequality holds

$$
\lambda_{i} \tau_{i}\left\|K_{i}(\hat{1})\right\|_{\infty}+\eta_{i} \theta_{i}\left\|\gamma_{i}\right\|_{\infty}<1 .
$$

Then the system (1.4) has at most the zero solution in $P_{I}$.

Proof. Suppose, for the sake of contradiction, there exists $u \in P_{I} \backslash\{0\}$ such that $u$ is a fixed point for $T+\Gamma$. Then there exists $j$ such that $\left\|u_{j}\right\|_{\infty}=\sigma>0$. Then we have

$$
\begin{aligned}
u_{j}(x)=\lambda_{j} K_{j} F_{j}(u)(x)+\eta_{j} h_{j}[u] \gamma_{j}(x) \leq\left\|\lambda_{j} K_{j} F_{j}(u)+\eta_{j} h_{j}[u] \gamma_{j}\right\|_{\infty} \\
\quad \leq\left\|\lambda_{j} K_{j}\left(\tau_{j} \sigma \hat{1}\right)\right\|_{\infty}+\left\|\eta_{j} \theta_{j} \sigma \gamma_{j}\right\|_{\infty}=\left(\lambda_{j} \tau_{j}\left\|K_{j}(\hat{1})\right\|_{\infty}+\eta_{j} \theta_{j}\left\|\gamma_{j}\right\|_{\infty}\right) \sigma<\sigma .
\end{aligned}
$$

By taking the supremum in (2.7) for $x \in \bar{\Omega}$ we obtain $\sigma<\sigma$, a contradiction.

\section{TWO EXAMPLES}

In the this last Section we show the applicability of results above in the context of systems of nonlocal elliptic equations with functional BCs. We begin by illustrating Theorem 2.3,

Example 3.1. Take $\Omega=\left\{x \in \mathbb{R}^{2}:\|x\|_{2}<1\right\}$ and consider the system

$$
\begin{cases}-\left(\int_{\Omega} e^{\left|\left(u_{1}, u_{2}\right)\right|} d x\right) \Delta u=\lambda_{1} e^{\left|\left(u_{1}, u_{2}\right)\right|}, & \text { in } \Omega, \\ -\left(\int_{\Omega} e^{u_{1}+u_{2}} d x\right) \Delta v=\lambda_{2}\left|\left(u_{1}, u_{2}\right)\right|^{2}, & \text { in } \Omega, \\ u_{1}=\eta_{1} h_{1}\left[\left(u_{1}, u_{2}\right)\right], \quad u_{2}=\eta_{2} h_{2}\left[\left(u_{1}, u_{2}\right)\right], & \text { on } \partial \Omega\end{cases}
$$

where $\left|\left(u_{1}, u_{2}\right)\right|=\max \left\{\left|u_{1}\right|,\left|u_{2}\right|\right\}$,

$$
h_{1}\left[\left(u_{1}, u_{2}\right)\right]=\left(u_{1}(0)\right)^{2}+\left(u_{2}(0)\right)^{\frac{1}{2}} \text { and } h_{2}\left[\left(u_{1}, u_{2}\right)\right]=\left(u_{1}(0)\right)^{\frac{1}{4}}+\left(\int_{\Omega} u_{2}(x) d x\right)^{2} .
$$

We wish to show that, under some algebraic conditions on the parameters $\lambda_{i}$ and $\eta_{i}$, the system 3.1 has a nonzero positive solution of norm less or equal to 1 .

First of all note that $K(\hat{1})=\frac{1}{4}\left(1-x_{1}^{2}-x_{2}^{2}\right)$, where $x=\left(x_{1}, x_{2}\right)$, and $\|K(\hat{1})\|_{\infty}=\frac{1}{4}$. We fix $\rho_{1}, \rho_{2}=1$ and consider

$$
\begin{aligned}
f_{1}\left(u_{1}, u_{2}, w_{1}\left[u, u_{2}\right]\right) & :=\lambda_{1} e^{\left|\left(u_{1}, u_{2}\right)\right|} w_{1}\left[u_{1}, u_{2}\right], \text { where } w_{1}\left[u_{1}, u_{2}\right]=\left(\int_{\Omega} e^{\left|\left(u_{1}, u_{2}\right)\right|} d x\right)^{-1}, \\
f_{2}\left(u_{1}, u_{2}, w_{2}\left[u, u_{2}\right]\right) & :=\lambda_{2}\left|\left(u_{1}, u_{2}\right)\right|^{2} w_{2}\left[u_{1}, u_{2}\right], \text { where } w_{2}\left[u_{1}, u_{2}\right]=\left(\int_{\Omega} e^{u_{1}+u_{2}} d x\right)^{-1} .
\end{aligned}
$$

In this case we have

$$
\left.\left[\underline{w}_{1}, \bar{w}_{1}\right]=\left[(e \pi)^{-1}, \pi^{-1}\right], \underline{w}_{8}, \bar{w}_{2}\right]=\left[\left(e^{2} \pi\right)^{-1}, \pi^{-1}\right]
$$


and therefore we get

$$
\begin{array}{ll}
M_{1}=f_{1}\left(1,1, \pi^{-1}\right)=e / \pi, & \bar{h}_{1} \leq 2, \\
M_{2}=f_{2}\left(1,1, \pi^{-1}\right)=1 / \pi, & \bar{h}_{2} \leq 1+\pi^{2} .
\end{array}
$$

Furthermore note that $f_{1}$ satisfies the condition $(c)$ in Theorem 2.3 for $\rho_{0}$ sufficiently small, due to the behaviour near the origin.

By Theorem 2.3 the system (3.1) has a nonzero positive solution $\left(u_{1}, u_{2}\right)$ such that $0<$ $\left\|\left(u_{1}, u_{2}\right)\right\| \leq 1$ for every $\lambda_{1}, \lambda_{2}, \eta_{1}, \eta_{2}>0$ with

$$
\frac{e \lambda_{1}}{4 \pi}+2 \eta_{1} \leq 1 \text { and } \frac{\lambda_{2}}{4 \pi}+\left(1+\pi^{2}\right) \eta_{2} \leq 1
$$

The inequality (3.2) is satisfied, for example, when $\lambda_{1}=1 / 3, \eta_{1}=1 / 4, \lambda_{2}=1 / 5, \eta_{2}=1 / 15$.

In this last example we illustrate the applicability of Theorem 2.4 .

Example 3.2. Take $\Omega=\left\{x \in \mathbb{R}^{2}:\|x\|_{2}<1\right\}$ and consider the system

$$
\begin{cases}-\left(\int_{\Omega} e^{\left|\left(u_{1}, u_{2}\right)\right|} d x\right) \Delta u=\lambda_{1}\left(u_{1}\right)^{2} \sin \left(u_{2}\right), & \text { in } \Omega, \\ -\left(\int_{\Omega} e^{u_{1}+u_{2}} d x\right) \Delta v=\lambda_{2}\left(u_{2}\right)^{4} \cos \left(u_{1}\right), & \text { in } \Omega, \\ u_{1}=\eta_{1} h_{1}\left[\left(u_{1}, u_{2}\right)\right], v=\eta_{2} h_{2}\left[\left(u_{1}, u_{2}\right)\right], & \text { on } \partial \Omega,\end{cases}
$$

where $h_{1}\left[\left(u_{1}, u_{2}\right)\right]=u_{1}(0)+\left(u_{2}(0)\right)^{2}$ and $h_{2}\left[\left(u_{1}, v\right)\right]=u_{1}(0)+\left(u_{2}(0)\right)^{3}$. As in Example 3.1 we set

$$
\begin{gathered}
f_{1}\left(u_{1}, u_{2}, w_{1}\left[u_{1}, u_{2}\right]\right):=\lambda_{1}\left(u_{1}\right)^{2} \sin \left(u_{2}\right) w_{1}\left[u_{1}, u_{2}\right], \text { where } w_{1}\left[u_{1}, u_{2}\right]=\left(\int_{\Omega} e^{\left|\left(u_{1}, u_{2}\right)\right|} d x\right)^{-1}, \\
f_{2}\left(u_{1}, u_{2}, w_{2}\left[u_{1}, u_{2}\right]\right):=\lambda_{2}\left(u_{2}\right)^{4} \cos \left(u_{1}\right) w_{2}\left[u_{1}, u_{2}\right], \text { where } w_{2}\left[u_{1}, u_{2}\right]=\left(\int_{\Omega} e^{u_{1}+u_{2}} d x\right)^{-1} .
\end{gathered}
$$

Fix $I=\left[0, \frac{\pi}{4}\right] \times\left[0, \frac{\pi}{2}\right]$ and note that in $\bar{\Omega} \times I$ we have

$$
0 \leq\left(u_{1}\right)^{2} \sin \left(u_{2}\right) \leq \frac{\pi}{4} u_{1}, 0 \leq\left(u_{2}\right)^{4} \cos \left(u_{1}\right) \leq \frac{\pi^{3}}{8} u_{2}
$$

Furthermore for $\left(u_{1}, u_{2}\right) \in P_{I}$, we have

$$
0 \leq h_{1}\left[\left(u_{1}, u_{2}\right)\right] \leq\left(\frac{\pi}{2}+1\right)\left\|\left(u_{1}, u_{2}\right)\right\|, 0 \leq h_{2}\left[\left(u_{1}, u_{2}\right)\right] \leq\left(\frac{\pi^{2}}{4}+1\right)\left\|\left(u_{1}, u_{2}\right)\right\| .
$$

Thus, in this case, the condition (2.6) is satisfied if we have

$$
\frac{1}{4 e} \lambda_{1}+\left(\frac{\pi}{2}+1\right) \eta_{1}<1 \text { and } \frac{\pi^{2}}{8 e^{2}} \lambda_{2}+\left(\frac{\pi^{2}}{4}+1\right) \eta_{2}<1
$$

Since the trivial solution is a solution of (3.3), as long as the inequality (3.4) is satisfied (for example when $\left.\lambda_{1}=1 / 2, \eta_{1}=1 / 3, \lambda_{2}=1 / 2, \eta_{2}=1 / 4\right)$, by Theorem 2.4 we obtain that the system (3.3) admits only the trivial solution in $P_{I}$. 


\section{ACKNOWLEDGEMENTS}

This manuscript was presented at the International Workshop on Nonlinear Dynamical Systems and Functional Analysis held in Brasilia (Brazil) in August 2018. G. Infante would like to thank the Workshop Organizers for their warm hospitality and generous support. G. Infante was partially supported by G.N.A.M.P.A. - INdAM (Italy).

\section{REFERENCES}

[1] H. Amann, Fixed point equations and nonlinear eigenvalue problems in ordered Banach spaces, SIAM. Rev., 18 (1976), 620-709.

[2] D. Arcoya, T. Leonori and A. Primo, Existence of solutions for semilinear nonlocal elliptic problems via a Bolzano theorem, Acta Appl. Math., 127 (2013), 87-104.

[3] G. Bonanno, Dirichlet problems without asymptotic conditions on the nonlinear term, Rend. Istit. Mat. Univ. Trieste, 49 (2017), 319-333.

[4] A. Cabada, An overview of the lower and upper solutions method with nonlinear boundary value conditions, Bound. Value Probl., (2011), Art. ID 893753, 18 pp.

[5] Y. Chen and H. Gao, Existence of positive solutions for nonlocal and nonvariational elliptic systems, Bull. Austral. Math. Soc., 72 (2005), 271-281.

[6] M. Chipot and F. J. S. A. Corrêa, Boundary layer solutions to functional elliptic equations, Bull. Braz. Math. Soc. (N.S.), 40 (2009), 381-393.

[7] M. Chipot and P. Roy, Existence results for some functional elliptic equations, Differential and Integral Equations, 27 (2014), 289-300.

[8] F. Cianciaruso, G. Infante and P. Pietramala, Solutions of perturbed Hammerstein integral equations with applications, Nonlinear Anal. Real World Appl., 33 (2017), 317-347.

[9] E. Caglioti, P.L. Lions, C. Marchioro and M. Pulvirenti, A special class of stationary flows for twodimensional Euler equations: a statistical mechanics description, Comm. Math. Phys., 143 (1992), $501-525$.

[10] R. Conti, Recent trends in the theory of boundary value problems for ordinary differential equations, Boll. Un. Mat. Ital., 22 (1967), 135-178.

[11] F. J. S. A. Corrêa, On positive solutions of nonlocal and nonvariational elliptic problems, Nonlinear Anal., 59 (2004), 1147-1155.

[12] F. J. S. A. Corrêa, S. D. B. Menezes and J. Ferreira; On a class of problems involving a nonlocal operator, Appl. Math. Comput., 147 (2004), 475-489.

[13] F. J. S. A. Corrêa and D. C. de Morais Filho, On a class of nonlocal elliptic problems via Galerkin method, J. Math. Anal. Appl., 310 (2005), 177-187.

[14] R. Enguiça and L. Sanchez, Radial solutions for a nonlocal boundary value problem, Bound. Value Probl., 2006, Art. ID 32950, 18 pp.

[15] P. Esposito, M. Grossi and A. Pistoia, On the existence of blowing-up solutions for a mean field equation, Ann. Inst. H. Poincaré Analyse Non Linéaire, 22 (2005) 227-257.

[16] F. Faraci and A. Iannizzotto, On the topological dimension of the solution set of a class of nonlocal elliptic problems, Topol. Methods Nonlinear Anal., 42 (2013), 1-8. 
[17] P. Fijałkowski and B. Przeradzki, On a radial positive solution to a nonlocal elliptic equation, Topol. Methods Nonlinear Anal., 21 (2003), 293-300.

[18] C. S. Goodrich, Perturbed Hammerstein integral equations with sign-changing kernels and applications to nonlocal boundary value problems and elliptic PDEs, J. Integral Equations Appl., 28 (2016), 509549.

[19] C. S. Goodrich, A new coercivity condition applied to semipositone integral equations with nonpositive, unbounded nonlinearities and applications to nonlocal BVPs, J. Fixed Point Theory Appl., 19 (2017), 1905-1938.

[20] C. S. Goodrich, New Harnack inequalities and existence theorems for radially symmetric solutions of elliptic PDEs with sign changing or vanishing Green's function, J. Differential Equations, 264 (2018), 236-262

[21] C. S. Goodrich, Radially symmetric solutions of elliptic PDEs with uniformly negative weight, Ann. Mat. Pura Appl., 197 (2018), 1585-1611.

[22] D. Guo and V. Lakshmikantham, Nonlinear problems in abstract cones, Academic Press, Boston, (1988).

[23] G. Infante, Nonzero positive solutions of a multi-parameter elliptic system with functional BCs, Topol. Methods Nonlinear Anal., 52 (2018), 665-675.

[24] G. L. Karakostas and P. Ch. Tsamatos, Existence of multiple positive solutions for a nonlocal boundary value problem, Topol. Methods Nonlinear Anal., 19 (2002), 109-121.

[25] G. L. Karakostas and P. Ch. Tsamatos, Multiple positive solutions of some Fredholm integral equations arisen from nonlocal boundary-value problems, Electron. J. Differential Equations, 2002, 17 pp.

[26] R. Jiang and C. Zhai, Properties of unique positive solutions for a class of nonlocal semilinear elliptic equations, Topol. Methods Nonlinear Anal., 50 (2017), 669-682.

[27] K. Q. Lan, Nonzero positive solutions of systems of elliptic boundary value problems, Proc. Amer. Math. Soc., 139 (2011), 4343-4349.

[28] K. Q. Lan, Existence of nonzero positive solutions of systems of second order elliptic boundary value problems J. Appl. Anal. Comput., 1 (2011), 21-31.

[29] R. Ma, A survey on nonlocal boundary value problems, Appl. Math. E-Notes, 7 (2007), 257-279.

[30] J. M. do Ó, S. Lorca, J. Sánchez and P. Ubilla, Positive solutions for some nonlocal and nonvariational elliptic systems, Complex Var. Elliptic Equ., 61 (2016), 297-314.

[31] T. F. Ma, Remarks on an elliptic equation of Kirchhoff type, Nonlinear Anal., 63 (2005), e1967-e1977.

[32] J. Morbach and F. J. S. A. Corrẽa, Some remarks on elliptic equations under nonlinear and nonlocal Neumann boundary conditions, Adv. Math. Sci. Appl., 23 (2013), 529-543.

[33] S. K. Ntouyas, Nonlocal initial and boundary value problems: a survey, Handbook of differential equations: ordinary differential equations. Vol. II, Elsevier B. V., Amsterdam, (2005), 461-557.

[34] C. V. Pao and Y. M. Wang, Nonlinear fourth-order elliptic equations with nonlocal boundary conditions, J. Math. Anal. Appl., 372 (2010), 351-365.

[35] M. Picone, Su un problema al contorno nelle equazioni differenziali lineari ordinarie del secondo ordine, Ann. Scuola Norm. Sup. Pisa Cl. Sci., 10 (1908), 1-95.

[36] C. A. Santos and L. Santos, How to break the uniqueness of $W_{\text {loc }}^{1, p}(\Omega)$-solutions for very singular elliptic problems by non-local terms, Z. Angew. Math. Phys., 69 (2018), Art. 145, 22 pp. 
[37] C. A. Santos, L. Santos and P. K. Mishra, Continuums of positive solutions for classes of nonautonomous and non-local problems with strong singular term, arXiv:1811.05051 [math.AP].

[38] R. Stanćzy, Nonlocal elliptic equations, Nonlinear Anal., 47 (2001), 3579-3584.

[39] A. Štikonas, A survey on stationary problems, Green's functions and spectrum of Sturm-Liouville problem with nonlocal boundary conditions, Nonlinear Anal. Model. Control, 19 (2014), 301-334.

[40] F. Wang and Y. An, Existence of nontrivial solution for a nonlocal elliptic equation with nonlinear boundary condition, Boundary Value Problems Volume 2009, Article ID 540360, 8 pages.

[41] J. R. L. Webb and G. Infante, Positive solutions of nonlocal boundary value problems: a unified approach, J. London Math. Soc., 74 (2006), 673-693.

[42] W. M. Whyburn, Differential equations with general boundary conditions, Bull. Amer. Math. Soc., 48 (1942), 692-704.

[43] B. Yan and T. Ma, The existence and multiplicity of positive solutions for a class of nonlocal elliptic problems, Bound. Value Probl. (2016) 2016:165.

[44] B. Yan and Q. Ren, Existence, uniqueness and multiplicity of positive solutions for some nonlocal singular elliptic problems, Electronic Journal of Differential Equations, 138 (2017), pp. 1-21

[45] B. Yan and D. Wang, The multiplicity of positive solutions for a class of nonlocal elliptic problem, $J$. Math. Anal. Appl., 442 (2016), 72-102. (2015), 951-967.

[46] E. Zeidler, Nonlinear functional analysis and its applications. I. Fixed-point theorems, Springer-Verlag, New York, (1986).

Gennaro Infante, Dipartimento di Matematica e Informatica, Università della Calabria, 87036 Arcavacata di Rende, Cosenza, Italy

E-mail address: gennaro.infante@unical.it 